\title{
MASSIV: Mass Assembly Survey with SINFONI in VVDS ${ }^{\star}$
}

\section{Evidence for positive metallicity gradients in $z \sim 1.2$ star-forming galaxies}

\author{
J. Queyrel ${ }^{1,2}$, T. Contini ${ }^{1,2}$, M. Kissler-Patig ${ }^{3}$, B. Epinat ${ }^{1,2}$, P. Amram ${ }^{4}$, B. Garilli ${ }^{5}$, O. Le Fèvre ${ }^{4}$, J. Moultaka ${ }^{1,2}$, \\ L. Paioro ${ }^{5}$, L. Tasca ${ }^{4}$, L. Tresse ${ }^{4}$, D. Vergani ${ }^{6}$, C. López-Sanjuan ${ }^{4}$, and E. Perez-Montero ${ }^{7}$
}

\author{
${ }^{1}$ Institut de Recherche en Astrophysique et Planétologie (IRAP), CNRS, 14 avenue Édouard Belin, 31400 Toulouse, France \\ e-mail: thierry.contini@irap.omp.eu \\ 2 IRAP, Université de Toulouse, UPS-OMP, Toulouse, France \\ 3 ESO, Karl-Schwarzschild-Str.2, 85748 Garching b. München, Germany \\ ${ }^{4}$ Laboratoire d'Astrophysique de Marseille, Université de Provence, CNRS, 38 rue Frédéric Joliot-Curie, 13388 Marseille Cedex 13, \\ France \\ 5 IASF-INAF, via Bassini 15, 20133 Milano, Italy \\ ${ }^{6}$ INAF-Osservatorio Astronomico di Bologna, via Ranzani 1, 40127 Bologna, Italy \\ 7 Instituto de Astrofísica de Andalucía - CSIC Apdo. 3004, 18080 Granada, Spain
}

Received 16 July 2011 / Accepted 14 November 2011

\section{ABSTRACT}

\begin{abstract}
Aims. The estimate of radial abundance gradients in high-redshift galaxies allows to constrain their star formation history and their interplay with the surrounding intergalactic medium.

Methods. We present VLT/SINFONI integral-field spectroscopy of a first sample of 50 galaxies at $z \sim 1.2$ in the MASSIV survey. Using the $\mathrm{N} 2$ ratio between the [ $\mathrm{N}$ II] 6584 and $\mathrm{H} \alpha$ rest-frame optical emission lines as a proxy for oxygen abundance in the interstellar medium, we measured the metallicity of the sample galaxies. We developed a tool to extract spectra in annular regions, leading to a spatially resolved estimate of the oxygen abundance in each galaxy. We were able to derive a metallicity gradient for 26 galaxies in our sample and discovered a significant fraction of galaxies with a "positive" gradient. Using a simple chemical evolution model, we derived infall rates of pristine gas onto the disks.

Results. Seven galaxies display a positive gradient at a high confidence level. Four out of these are interacting, and one is a chain galaxy. We suggest that interactions might be responsible for shallowing and even inverting the abundance gradient. We also identify two interesting correlations in our sample: a) galaxies with higher gas velocity dispersion have shallower/positive gradients; and b) metal-poor galaxies tend to show a positive gradient, whereas metal-rich ones tend to show a negative one. This last observation can be explained by the infall of metal-poor gas into the center of the disks. We address the question of the origin of this infall under the influence of gas flows triggered by interactions and/or cold gas accretion. All the data published in this paper are publicly available at the time of publication following this link: http://cosmosdb. lambrate.inaf.it/VVDS-SINFONI.
\end{abstract}

Key words. galaxies: evolution - galaxies: abundances - galaxies: high-redshift

\section{Introduction}

A key goal for galaxy evolution and formation models is to understand the different mechanisms of galaxy assembly at various cosmic epochs. In this context, the gas phase and stellar metallicities have proven to be important parameters in constraining the star formation history of galaxies.

The relation between galaxy mass and metallicity, which was first discovered by Lequeux et al. (1979) in irregular galaxies, exists for star-forming galaxies both in the nearby universe (Tremonti et al. 2004; Lamareille et al. 2004) and at high redshifts $z \sim 0.7-3$ (Lamareille et al. 2006, 2009; Pérez-Montero et al. 2009; Queyrel et al. 2009; Erb et al. 2006; Maiolino et al. 2008; Mannucci et al. 2009). This trend by more massive galaxies toward higher gas-phase metallicity can be explained by

* This work is based on observations collected at the European Southern Observatory (ESO) Very Large Telescope, Paranal, Chile, as part of the Programs 179.A-0823, 78.A-0177, and 75.A-0318. This work also benefits from data products produced at TERAPIX and the Canadian Astronomy Data Centre as part of the Canada-FranceHawaii Telescope Legacy Survey, a collaborative project of NRC and the CNRS. various related properties: gas inflows and/or outflows, strength of the gravitational potential, efficiency of the star formation depending on stellar mass, etc. Their relative importance is currently being explored in numerical simulations.

Studying the abundance in $\mathrm{HII}_{\mathrm{II}}$ regions of nearby spiral galaxies has unveiled metallicity gradients in the local Universe (Pagel \& Edmunds 1981; Vila-Costas \& Edmunds 1992; Considère et al. 2000; Pilyugin et al. 2004). In $z \sim 0$ galaxies, the metallicity generally decreases from the center to the outskirts (up to $\Delta_{r} Z \sim-0.1 \mathrm{dex} \mathrm{kpc}^{-1}$ ). The strength of these gradients seems to correlate with various parameters such as rotation velocity, morphological type, luminosity, and the presence of a bar. Different physical processes have been proposed to explain these patterns, including radial gas-flows (Koeppen 1994), self-regulating star formation (Phillipps \& Edmunds 1991), variable yields (Vila-Costas \& Edmunds 1992), or continuous infall of gas onto the disk (Magrini et al. 2007).

Merging events - which are believed to play a substantial role in galaxy evolution (e.g. de Ravel et al. 2009; López-Sanjuan et al. 2011) - seem to be a key physical process in shaping the metallicity gradients of interacting galaxies (Rupke et al. 2010). Recent observations have suggested that 
galaxies involved in merging events show lower nuclear metallicities that are caused by the infall of pristine gas into the nucleus. Merging events could also account for outliers to the mass-metallicity relation (Kewley et al. 2006; Michel-Dansac et al. 2008; Peeples et al. 2009; Queyrel et al. 2009; Alonso et al. 2010; Montuori et al. 2010; Kewley et al. 2010).

Metallicity gradients have been observed in the stellar populations of early-type galaxies as well. They appear to correlate with macroscopic properties such as stellar mass (e.g. Spolaor et al. 2009). It has been demonstrated that a metallicity gradient in the stellar populations could survive a major merger event, although it will be weakened. These observations contrast the predictions of the simple monolithical collapse scenario (White 1980; Bekki \& Shioya 1999; Kobayashi 2004; Di Matteo et al. 2009).

In nearby galaxies, metallicity gradients can be inferred from observation of different emission-line ratios (in $\mathrm{H}$ II regions) or from stellar absorption lines. In the distant universe $(z \gtrsim 0.5)$, low signal-to-noise ratio (hereafter $S N R)$ and low spatial-resolution data make these measurements much more challenging. However, thanks to powerful integral-field spectrographs mounted on the largest telescopes, it is now possible to determine spatially resolved physical parameters of highredshift galaxies.

Many studies have taken advantage of this technique to study the gas dynamics of distant galaxies, from $z \sim 0.6$ (Flores et al. 2006) up to $z \gtrsim 2$ (Förster Schreiber et al. 2009; Law et al. 2009; Epinat et al. 2009; Gnerucci et al. 2011). Recently, the Mass Assembly Survey with SINFONI in VVDS (MASSIV; Contini et al. 2012) has collected data of $z \sim 1-2$ galaxies observed with the integral-field spectrograph SINFONI at the VLT (Eisenhauer et al. 2003). Depending on the galaxy redshift, the bright $\mathrm{H} \alpha$ emission line is targeted in the $J$ or $H$ band. The nitrogen line [ $\left.\mathrm{N}_{\mathrm{II}}\right] 6584$ close to $\mathrm{H} \alpha$ allows estimation of the gasphase metallicity via the $\mathrm{N} 2$ ratio $(\mathrm{N} 2=\log ([\mathrm{N}$ II $] 6584 / \mathrm{H} \alpha))$ and the corresponding abundance calibration (Kewley \& Dopita 2002; Denicoló et al. 2002; Pérez-Montero \& Contini 2009).

The aim of this paper is to investigate - for the first time at a redshift around 1 - metallicity gradients of 50 star-forming galaxies in the MASSIV sample. To achieve this goal, we have developed a dedicated program to analyze the low SNR data cubes. Two companion papers (Vergani et al. 2012; Epinat et al. 2012) discuss the associated fundamental scaling relations (e.g. the Tully-Fischer relation for disks) and the kinematical properties of the sample galaxies. The results of these three studies are cross-correlated in order to investigate possible relations between the metallicity gradients and global properties of galaxies at $z \sim 1.2$.

The paper is organized as follows. In Sect. 2 we briefly summarize the properties of the galaxy sample, the data reduction technique, the emission-line measurements, the level of active galactic nucleus (AGN) contamination, the kinematics, and the mass (both stellar and dark matter halo) estimates. In Sect. 3 we present and discuss the determined metallicity gradients. Our conclusions are drawn in Sect. 4.

Throughout the paper, we assume a $\Lambda$ CDM cosmology with $\Omega_{\mathrm{m}}=0.3, \Omega_{\Lambda}=0.7$ and $H_{0}=70 \mathrm{~km} \mathrm{~s}^{-1} \mathrm{Mpc}^{-1}$.

\section{Data description}

\subsection{Target selection, observations, and data reduction}

The galaxy sample studied in this paper is the "first epoch" sample of the MASSIV project (ESO Large Program,
PI.: T. Contini). A full description of the sample can be found in Contini et al. (2012). We briefly summarize some properties of this sample of 50 galaxies below.

The galaxies were selected from the VIMOS VLT Deep Survey (VVDS) in the deep $\left(I_{\mathrm{AB}}<24\right.$; Le Fèvre et al. 2005), ultra-deep $\left(I_{\mathrm{AB}}<24.75\right.$; Le Fèvre et al., in prep. $) \mathrm{RA}=02 \mathrm{~h}$, and wide $\left(I_{\mathrm{AB}}<22.5\right.$; Garilli et al. 2008) RA $=14 \mathrm{~h}, 22 \mathrm{~h}$ fields. The galaxies were chosen according to their redshift such that their $\mathrm{H} \alpha$ line (or [O III] 5007 in a few cases) was visible in the $J$ or $H$ band, and was not affected by a bright $\mathrm{OH}$ skyline. Galaxies were selected to be star-forming on the basis of their [O II] 3727 emission line strength. The observations were performed between April 2007 and August 2009. The general properties (RA, Dec, redshift, I-band magnitude) and characteristic observational configuration (spectral band, adaptive optics or not, exposure time, spatial resolution) of each galaxy are gathered in Table 1. Most of the galaxies (85\%) in this "first epoch" sample were observed in a seeing-limited mode (with a spatial sampling of $\left.0.125^{\prime \prime}\right)$. However, seven galaxies were acquired with adaptive optics assisted with a laser guide star (AO/LGS, 0.05 " spatial sampling). Among the 50 "first epoch" MASSIV galaxies observed with SINFONI, four galaxies have not been detected (see Epinat et al. 2012, for details).

The data reduction was performed with the ESO SINFONI pipeline, using the standard master calibration files provided by ESO. The absolute astrometry for the SINFONI data cubes was derived from nearby bright stars that were also used for PSF measurements. Custom IDL and Python scripts were used to flux calibrate, align, and combine all the individual exposures. For each galaxy a cube that was not sky-subtracted was also created, mainly to estimate the effective spectral resolution. For more details on data reduction, we refer to Epinat et al. (2012).

The $I$-band images for all the galaxies were obtained through CFHT Megacam imaging (for the $22 \mathrm{~h}$ and $02 \mathrm{~h}$ fields, from the CFHTLS "best seeing") and CFHT-12K imaging (for the $14 \mathrm{~h}$ field). These images were used for two purposes: (i) refining the SINFONI astrometry with the relative position of the PSF star, and (ii) deriving morphological parameters (used as input to the kinematics modeling, see Epinat et al. 2012).

\subsection{Measurement of emission lines}

As mentioned in the previous section, galaxies were selected as star-forming such that emission lines from $\mathrm{H}$ in regions are visible in the near infrared. Given the redshift range of MASSIV galaxies, we targeted, in most of the cases, the rest-frame optical $\mathrm{H} \alpha$ emission line in the $J$ (for $z<1.2$ ) or $H$ (for $z>1.2$ ) band. For four objects (VVDS020126402, 020167131, 020306817, and 220148046) we observed the [O III]5007 line, which allowed us to increase the redshift window. Two of them were not detected. These galaxies are not considered in this paper as we cannot derive any metallicity from the [O III $] 5007$ emission line alone. In the former cases, we took advantage of the proximity of the nitrogen line to $\mathrm{H} \alpha$ to derive the metallicity of a majority of the galaxies using the $\left[\mathrm{N}_{\mathrm{II}}\right] 6584 / \mathrm{H} \alpha$ line ratio as a proxy for oxygen abundance (see Sect. 3).

\subsubsection{Integrated fluxes}

The global integrated flux of each emission line was calculated using a mask designed for each galaxy. The flux was collected over a region where the signal-to-noise ratio of the $\mathrm{H} \alpha$ line is above a fixed threshold of $S N R>2$. The $S N R$ maps were 
J. Queyrel et al.: MASSIV: Mass Assembly Survey with SINFONI in VVDS. III.

Table 1. General properties and SINFONI observing log of the 50 "first epoch" galaxies of MASSIV.

\begin{tabular}{|c|c|c|c|c|c|c|c|c|}
\hline Galaxy & $\begin{array}{c}\text { RA (J2000) } \\
\text { [deg] } \\
(2)\end{array}$ & $\begin{array}{c}\text { Dec }(\mathrm{J} 2000) \\
\text { [deg] } \\
\text { (3) }\end{array}$ & Band & AO/LGS & $\begin{array}{l}t_{\text {exp }} \\
{[\mathrm{s}]} \\
(6)\end{array}$ & $\begin{array}{c}I_{\mathrm{AB}} \\
{[\mathrm{mag}]} \\
(7)\end{array}$ & $\begin{array}{c}\text { Mean PSF } \\
{\left[{ }^{\prime \prime}\right]} \\
(8)\end{array}$ & $z_{\text {SINFONI }}$ \\
\hline 020106882 & 36.340833 & -4.771834 & $H$ & 0 & 4800 & 23.18 & 0.49 & 1.3991 \\
\hline 020116027 & 36.463055 & -4.751243 & $H$ & 0 & 4500 & 22.88 & 0.60 & 1.5302 \\
\hline 020126402 & 36.298576 & -4.727812 & $J$ & 1 & 3600 & 22.99 & & 1.2332 \\
\hline 020147106 & 36.689110 & -4.679830 & $H$ & 0 & 7200 & 22.51 & 0.65 & 1.5195 \\
\hline 020149061 & 36.771769 & -4.674816 & $H$ & 0 & 4800 & 22.56 & 0.85 & 1.2905 \\
\hline 020164388 & 36.712244 & -4.639131 & $H$ & 0 & 4800 & 22.51 & 0.83 & 1.3547 \\
\hline 020167131 & 36.697114 & -4.632054 & $J$ & 0 & 7200 & 23.01 & 0.68 & $1.2246^{*}$ \\
\hline 020182331 & 36.684314 & -4.597755 & $H$ & 0 & 10800 & 22.73 & 0.74 & 1.2290 \\
\hline 020193070 & 36.327984 & -4.572212 & $J$ & 0 & 7200 & 23.41 & 0.58 & 1.0279 \\
\hline 020208482 & 36.319735 & -4.536649 & $J$ & 0 & 7200 & 23.25 & 0.58 & 1.0375 \\
\hline 020214655 & 36.597688 & -4.523057 & $J$ & 0 & 4800 & 23.05 & 0.87 & 1.0395 \\
\hline 020217890 & 36.613174 & -4.514397 & $H$ & 0 & 7200 & 23.99 & & 1.5129 \\
\hline 020239133 & 36.679087 & -4.475228 & $J$ & 0 & 4800 & 22.85 & 0.79 & 1.0194 \\
\hline 020240675 & 36.725581 & -4.471541 & $H$ & 0 & 4800 & 23.45 & 0.85 & 1.3270 \\
\hline 020255799 & 36.691075 & -4.437713 & $J$ & 0 & 4800 & 23.63 & 0.76 & 1.0351 \\
\hline 020261328 & 36.796038 & -4.425444 & $H$ & 0 & 3600 & 23.90 & 0.62 & 1.5290 \\
\hline 020278667 & 36.492598 & -4.386738 & $J$ & 0 & 7200 & 23.25 & 0.65 & 1.0516 \\
\hline 020283083 & 36.628634 & -4.376830 & $H$ & 0 & 4800 & 23.07 & 0.78 & 1.2818 \\
\hline 020283830 & 36.620565 & -4.375444 & $H$ & 0 & 7200 & 22.92 & 0.77 & 1.3949 \\
\hline 020294045 & 36.446413 & -4.352110 & $J$ & 0 & 7200 & 22.80 & 0.59 & 1.0028 \\
\hline 020306817 & 36.459649 & -4.323037 & $J$ & 0 & 7200 & 23.29 & & 1.2225 \\
\hline 020363717 & 36.598723 & -4.199505 & $H$ & 0 & 4800 & 22.61 & 0.64 & 1.3339 \\
\hline 020370467 & 36.561244 & -4.184841 & $H$ & 0 & 4800 & 23.46 & 0.71 & 1.3338 \\
\hline 020386743 & 36.808285 & -4.149879 & $J$ & 0 & 7200 & 22.58 & 0.73 & 1.0487 \\
\hline 020461235 & 36.696290 & -4.398832 & $J$ & 0 & 7200 & 22.64 & 0.63 & 1.0349 \\
\hline 020461893 & 36.801068 & -4.386450 & $J$ & 0 & 4800 & 23.45 & 0.60 & 1.0486 \\
\hline 020465775 & 36.747379 & -4.316665 & $H$ & 0 & 4800 & 23.15 & 0.88 & 1.3583 \\
\hline 140083410 & 209.460955 & 4.294251 & $J$ & 0 & 4800 & 21.82 & 0.69 & 0.9435 \\
\hline 140096645 & 209.609695 & 4.329940 & $J$ & 0 & 7200 & 22.28 & 0.56 & 0.9655 \\
\hline 140123568 & 208.990111 & 4.405582 & $J$ & 0 & 7200 & 23.43 & 0.76 & 1.0012 \\
\hline 140137235 & 209.053257 & 4.442166 & $J$ & 0 & 4800 & 22.38 & 0.76 & 1.0445 \\
\hline 140217425 & 209.485039 & 4.643635 & $J$ & 0 & 6000 & 21.58 & 0.95 & 0.9792 \\
\hline 140258511 & 210.081944 & 4.746065 & $H$ & 0 & 4800 & 21.17 & 0.49 & 1.2423 \\
\hline 140262766 & 209.981154 & 4.758375 & $H$ & 0 & 7200 & 23.68 & 0.51 & 1.2836 \\
\hline 140545062 & 209.898275 & 5.508636 & $J$ & 0 & 7200 & 22.42 & 0.70 & 1.0408 \\
\hline 220014252 & 334.440374 & 0.477630 & $H$ & 0 & 7200 & 22.04 & 0.70 & 1.3105 \\
\hline 220015726 & 333.926894 & 0.484332 & $H$ & 0 & 7200 & 22.42 & 0.46 & 1.2933 \\
\hline 220071601 & 334.506537 & 0.759637 & $H$ & 1 & 4800 & 21.74 & & 1.3538 \\
\hline 220148046 & 333.657514 & 1.139144 & $H$ & 1 & 4800 & 22.39 & 0.27 & $2.2441^{*}$ \\
\hline 220376206 & 335.024084 & -0.139356 & $H$ & 0 & 7200 & 21.78 & 0.50 & 1.2445 \\
\hline 220386469 & 334.985762 & -0.050878 & $J$ & 1 & 2400 & 22.10 & 0.23 & 1.0226 \\
\hline 220397579 & 335.152164 & 0.029634 & $J$ & 0 & 7200 & 22.42 & 0.64 & 1.0379 \\
\hline 220544103 & 333.857118 & 0.110982 & $H$ & 0 & 7200 & 22.38 & 0.76 & 1.3973 \\
\hline 220544394 & 333.600634 & 0.113027 & $J$ & 0 & 7200 & 22.15 & 0.58 & 1.0101 \\
\hline 220576226 & 334.047658 & 0.275121 & $J$ & 0 & 7200 & 21.80 & 0.58 & 1.0217 \\
\hline 220578040 & 334.267094 & 0.282327 & $J$ & 0 & 7200 & 22.30 & 0.62 & 1.0462 \\
\hline 220584167 & 333.845992 & 0.313059 & $H$ & 0 & 7200 & 21.96 & 0.75 & 1.4655 \\
\hline 220596913 & 333.621601 & 0.371914 & $H$ & 1 & 7200 & 21.80 & 0.18 & 1.2658 \\
\hline 910193711 & 36.442825 & -4.542796 & $H$ & 1 & 4800 & 22.69 & 0.27 & 1.5564 \\
\hline 910279515 & 36.401024 & -4.354381 & $H$ & 1 & 4800 & 23.71 & 0.21 & 1.4013 \\
\hline
\end{tabular}

Notes. Column 1: identifier in the VVDS catalog, Cols. 2 and 3: RA \& Dec in degrees, Col. 4: spectral band of the observation, Col. 5: seeinglimited mode (0) or Adaptive Optics (1), Col. 6: on-source total exposure time, Col. 7: I-band magnitude in the AB system, Col. 8: mean spatial resolution (PSF) as measured on a nearby star, Col. 9: redshift from $\mathrm{H} \alpha$ (or [O III] 5007 when marked with an asterisk) as measured with SINFONI. For the four galaxies (VVDS020126402, VVDS020217890, VVDS020306817, and VVDS220071601) which have not been detected with SINFONI, redshift comes from VIMOS spectra.

produced using a Gaussian-fitting procedure written in IDL, already used and described in Epinat et al. (2012). A Gaussian spatial smoothing of $2 \times 2$ spaxels $\left(0.25^{\prime \prime} \times 0.25^{\prime \prime}\right)$ was used, thereby increasing the $S N R$ without degrading the final spatial resolution $\left(\sim 0.65^{\prime \prime}\right.$ on average). The line-position map produced by this procedure was used to shift in wavelength every single spectrum so as to compensate for the Doppler effect corresponding to the measured $\mathrm{H} \alpha$ velocity at that position. In this way, the broadening of the line by large-scale rotation in the spatially-integrated spectrum was largely removed, and the line profile fitted with a Gaussian more easily, increasing the accuracy of line measurements. 
The $\mathrm{H} \alpha$ fluxes were measured on flux-calibrated integrated spectra using a Gaussian fit and a flat continuum. These fluxes were corrected for dust reddening using the extinction coefficient derived from the SED fitting (see Contini et al. 2012; Vergani et al. 2012). The line ratios, on the other hand, were determined from the integrated spectra in counts. Indeed, the flux calibration procedure would have added noise on top of the already low SNR data. Furthermore, the lines of interest (namely, $\mathrm{H} \alpha$ and $[\mathrm{N}$ II]6584) are close enough in wavelength to assume that the sensitivity curve is constant over the wavelength range of interest. The same argument justifies not correcting the emission-line ratios for differential extinction. Table 2 lists our final integrated $\mathrm{H} \alpha$ flux, $\mathrm{N} 2$ emission line ratio $\left(N 2=\log \left(\left[\mathrm{N}_{\mathrm{II}}\right] 6584 / \mathrm{H} \alpha\right)\right)$, and star formation rates (SFR, corrected or not for dust reddening). Among the 44 galaxies detected in $\mathrm{H} \alpha$ with SINFONI, we were able to measure the [N $\mathrm{NI}] 6584$ emission-line in 34 galaxies and from that derive integrated $\mathrm{N} 2$ emission-line ratio and metallicity for these objects.

\subsubsection{Spatially-resolved flux and line ratio}

For many galaxies in our sample, the low SNR of the data cubes prevents us from measuring with high confidence the emission lines surrounding $\mathrm{H} \alpha$ in each spaxel: namely [ $\left.\mathrm{N}_{\text {II }}\right] 6584$, [S II]6717,31, and [O I 6300 , which is detected only in one galaxy: VVDS140096645. These lines might not be bright enough to be detected, or a bright $\mathrm{OH}$ sky-line is too close. To increase the $S N R$ of our final spectra, we summed the spectra of the spaxels gathered in a specified region, and measured the line fluxes in the resulting spectra.

We developed a specific Python procedure to do this analysis within our data cubes. The program allowed defining a region spaxel-by-spaxel or defining a region with contours on 2D maps (such as $\mathrm{H} \alpha$ flux or SNR maps). In a given region, the spaxelto-spaxel line shift due to the rotation velocity of the gas along the line-of-sight was fully corrected for (see Sect. 2.2.1). The integrated spectrum in a defined region was then fitted with a flat continuum and two Gaussians, one for $\mathrm{H} \alpha$ and one for [ $\left.\mathrm{N}_{\text {II }}\right] 6584$ (the sulfur doublet was neglected for this analysis as it was only detected in a few cases). The width of each Gaussian was set to be the same as the emission of the collisional and recombination lines traces the same ionized gas in the galaxies. The fit was weighted using the corresponding non sky-subtracted spectrum, i.e. giving a lower weight to the channels affected by the subtraction of a strong sky-line. We then estimated the $1 \sigma$ error on the fluxes with a Monte-Carlo technique, in which the fit was considered to be a "noise-free" model, to which we added a Gaussian noise with a standard deviation corresponding to the residuals from the initial spectrum minus the "noise-free" model. This operation was repeated a hundred times, which gave a set of parameters on which the $1 \sigma$ deviation was computed. Figure 1 shows a result of our fits in different regions for two galaxies.

Finally, we estimated spatially-resolved $\mathrm{H} \alpha$ and [N II]6584 emission-line flux for $26 / 34(\sim 75 \%)$ galaxies in the sample. The remaining eight galaxies either have too low $S N R$, or the [N II]6584 emission-line is polluted by sky-line residuals.

\subsection{AGN contamination}

A recent study (Wright et al. 2010) has shown that unveiling the presence of AGN in high-redshift galaxies is a difficult exercise. In the case of metallicity studies, in which abundances are deduced from the ratio of different emission lines of the
Table 2. Emission-line measurements and star formation rates.

\begin{tabular}{|c|c|c|c|c|}
\hline Galaxy & $\begin{array}{r}F(\mathrm{H} \alpha) \\
{\left[10^{-17} \mathrm{erg}^{-1} \mathrm{~cm}^{-2}\right]}\end{array}$ & $\mathrm{N} 2$ & $\begin{array}{r}S F R_{\mathrm{H} \alpha} \\
{\left[M_{\odot} \mathrm{yr}^{-1}\right]}\end{array}$ & $\begin{array}{r}S F R_{\mathrm{H} \alpha}^{\mathrm{cor}} \\
{\left[M_{\odot} \mathrm{yr}^{-1}\right]}\end{array}$ \\
\hline (1) & $(2)$ & (3) & (4) & (5) \\
\hline 020106882 & $14.0 \pm 0.8$ & $-0.71 \pm 0.12$ & 13.3 & 38.1 \\
\hline 020116027 & $12.4 \pm 2.9$ & $-1.02 \pm 0.15$ & 14.7 & 42.2 \\
\hline 020126402 & & & & \\
\hline 020147106 & $46.8 \pm 5.8$ & $-0.95 \pm 0.14$ & 54.3 & 92.1 \\
\hline 020149061 & $20.6 \pm 1.6$ & & 16.0 & 45.9 \\
\hline 020164388 & $24.1 \pm 1.3$ & $-0.86 \pm 0.10$ & 21.1 & 46.5 \\
\hline 020167131 & & & & \\
\hline 020182331 & $15.2 \pm 5.0$ & & 10.5 & 51.1 \\
\hline 020193070 & $6.7 \pm 0.9$ & $-0.56 \pm 0.13$ & 3.0 & 14.4 \\
\hline 020208482 & $2.6 \pm 0.5$ & & 1.2 & 2.6 \\
\hline 020214655 & $23.3 \pm 1.1$ & $-0.68 \pm 0.06$ & 10.6 & 51.7 \\
\hline 020217890 & & $\ldots$ & & \\
\hline 020239133 & $7.8 \pm 1.0$ & 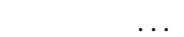 & 3.4 & 16.5 \\
\hline 020240675 & $8.5 \pm 1.0$ & & 7.1 & 15.7 \\
\hline 020255799 & $5.8 \pm 0.7$ & $-0.65 \pm 0.15$ & 2.6 & 12.8 \\
\hline 020261328 & $9.5 \pm 0.9$ & $-1.06 \pm 0.48$ & 11.2 & 19.0 \\
\hline 020278667 & $2.4 \pm 1.3$ & & 1.1 & 4.2 \\
\hline 020283083 & $13.4 \pm 1.0$ & $-0.70 \pm 0.12$ & 10.3 & 17.4 \\
\hline 020283830 & $11.0 \pm 1.1$ & $-0.82 \pm 0.17$ & 10.3 & 50.4 \\
\hline 020294045 & $15.5 \pm 2.8$ & $\ldots$ & 6.5 & 14.3 \\
\hline 020306817 & & & $\ldots$ & \\
\hline 020363717 & $37.4 \pm 4.3$ & $-0.87 \pm 0.09$ & 31.5 & 53.4 \\
\hline 020370467 & $17.3 \pm 2.9$ & $-0.69 \pm 0.11$ & 14.6 & 71.2 \\
\hline 020386743 & $17.4 \pm 1.7$ & $-0.91 \pm 0.08$ & 8.1 & 39.4 \\
\hline 020461235 & $7.5 \pm 2.0$ & $-0.67 \pm 0.12$ & 3.3 & 9.6 \\
\hline 020461893 & $14.5 \pm 1.2$ & $\ldots$ & 6.7 & 14.9 \\
\hline 020465775 & $14.5 \pm 2.8$ & & 12.8 & 62.2 \\
\hline 140083410 & $47.0 \pm 3.3$ & $-0.72 \pm 0.08$ & 16.8 & 37.1 \\
\hline 140096645 & $55.4 \pm 4.2$ & $-0.25 \pm 0.02$ & 20.9 & 102.1 \\
\hline 140123568 & $5.8 \pm 1.2$ & $-0.69 \pm 0.10$ & 2.4 & 11.8 \\
\hline 140137235 & $9.6 \pm 2.3$ & $-0.79 \pm 0.13$ & 4.4 & 21.6 \\
\hline 140217425 & $104.7 \pm 4.9$ & $-0.44 \pm 0.02$ & 41.0 & 200.0 \\
\hline 140258511 & $42.5 \pm 2.7$ & $-0.51 \pm 0.10$ & 30.0 & 146.4 \\
\hline 140262766 & $13.1 \pm 5.0$ & $-0.75 \pm 0.15$ & 10.0 & 10.0 \\
\hline 140545062 & $29.4 \pm 3.3$ & $-0.75 \pm 0.06$ & 13.4 & 22.7 \\
\hline 220014252 & $50.3 \pm 3.1$ & $-0.67 \pm 0.08$ & 40.5 & 197.5 \\
\hline 220015726 & $47.5 \pm 6.7$ & $-0.69 \pm 0.04$ & 37.0 & 106.4 \\
\hline 220071601 & $\ldots$ & $\ldots$ & $\ldots$ & $\ldots$ \\
\hline 220148046 & & & $\ldots$ & \\
\hline 220376206 & $72.4 \pm 5.1$ & $-1.04 \pm 0.10$ & 51.2 & 249.7 \\
\hline 220386469 & $17.1 \pm 2.4$ & $-1.14 \pm 0.16$ & 7.5 & 36.5 \\
\hline 220397579 & $65.1 \pm 9.1$ & $-1.07 \pm 0.07$ & 29.4 & 143.2 \\
\hline 220544103 & $55.0 \pm 1.9$ & $-0.56 \pm 0.09$ & 52.2 & 117.5 \\
\hline 220544394 & $24.3 \pm 1.7$ & $-0.89 \pm 0.07$ & 10.3 & 50.1 \\
\hline 220576226 & $31.2 \pm 1.6$ & $-0.69 \pm 0.04$ & 13.6 & 66.3 \\
\hline 220578040 & $20.5 \pm 1.9$ & $-0.80 \pm 0.07$ & 9.4 & 20.8 \\
\hline 220584167 & $64.3 \pm 1.3$ & $-0.91 \pm 0.10$ & 68.6 & 202.6 \\
\hline 220596913 & $30.0 \pm 1.0$ & & 23.2 & 36.1 \\
\hline 910193711 & $32.9 \pm 18.4$ & $-0.68 \pm 0.09$ & 40.6 & 197.7 \\
\hline 910279515 & $14.9 \pm 2.1$ & $-0.83 \pm 0.21$ & 14.2 & 69.0 \\
\hline
\end{tabular}

Notes. Column 2: $\mathrm{H} \alpha$ flux. Column 3: $N 2=\log \left(\left[\mathrm{N}_{\text {II }}\right] 6584 / \mathrm{H} \alpha\right)$ emission-line ratio. Columns 4 and 5: $\mathrm{H} \alpha$-based star formation rates (corrected - and not - for dust reddening).

ionized gas, it is critical to check that the intensity and width of these lines come from star formation and are not related to any non thermal nuclear activity. The common way to disentangle AGN contribution from star-forming galaxies consists in comparing the relative intensity of the main nebular emission lines (mainly [O III] 5007, $\mathrm{H} \beta, \mathrm{H} \alpha$, and [N II]6584) in a diagnostic diagram, a so-called BPT diagram (Baldwin et al. 1981; Kewley et al. 2001). Various physical conditions in the ISM - SFR, 

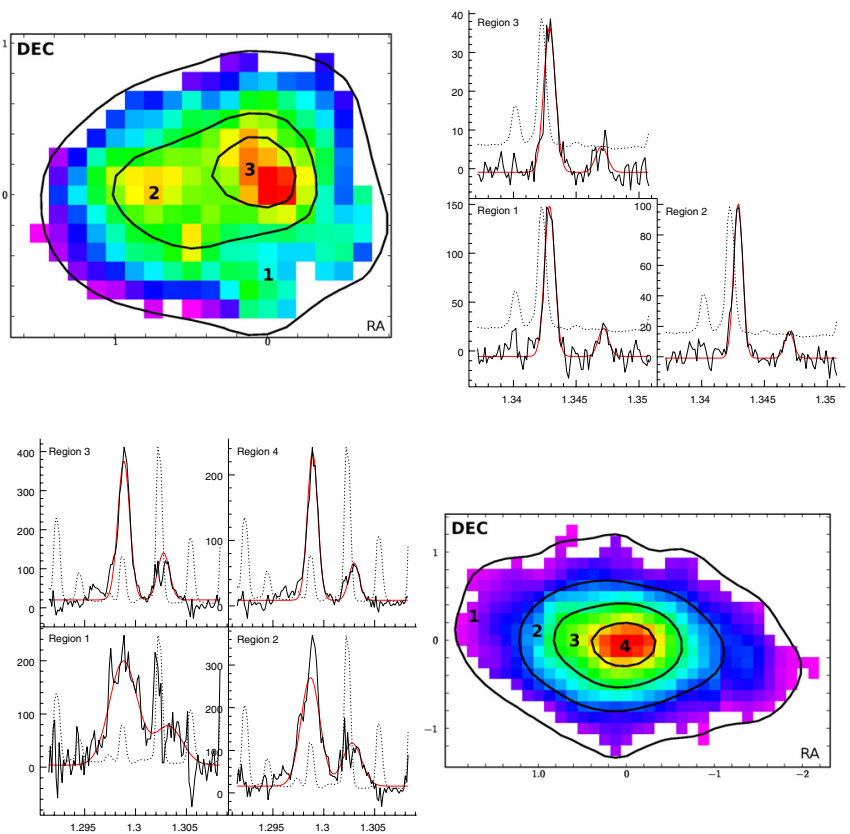

Fig. 1. Examples of spatially-resolved emission-lines measurements in two MASSIV galaxies (top: VVDS220578040, bottom: VVDS140217425). Color-coded images show the regions defined with $\mathrm{H} \alpha$ contours used to derive the metallicity gradients. The 1D spectra integrated over the different regions are shown with solid black lines. Model (in red) and sky (dashed line) spectra are also shown. The sky spectrum is used as a weight in the fitting procedure. Region 1 is the farthest out with the region number increasing towards the center.

ionization parameter, metallicity, and/or chemical composition - have been invoked to explain that some high-z star-forming galaxies lie in the transition region of the local BPT diagram (as defined by the SDSS galaxies) between star-forming galaxies and AGN hosts. Wright et al. (2010) have been able - thanks to high resolution adaptive optics observations - to subtract the active nuclear emission in a $z \sim 1.6$ galaxy (HDF-BMZ1299), and have shown that the residual extended star-forming emission was characteristic of a local SDSS star-forming galaxy, whereas the integrated emission would have placed the object in the transition region.

The nature of our observations did not give us simultaneous access to the set of emission lines ([O III] $] 5007, \mathrm{H} \beta, \mathrm{H} \alpha$, and $\left[\mathrm{N}_{\text {II }}\right] 6584$ or $\left.\left[\mathrm{S}_{\text {II }}\right] 6717,31\right)$ commonly used in standard diagnostic diagrams. However, for all but two objects in our sample, the emission-line ratio $\mathrm{N} 2=\log \left(\left[\mathrm{N}_{\mathrm{II}}\right] 6584 / \mathrm{H} \alpha\right)$ is lower than -0.5 , with a median $\mathrm{N} 2$ value of -0.72 . Such low values are indicative of a very low contamination by AGN in our sample (e.g. Baldwin et al. 1981).

For 24 galaxies of our sample we calculated, following Wright et al. (2010), the N2 "concentrated ratio", corresponding to the value in the nuclear region of the galaxy. We defined the nuclear region as the spaxel with the highest $\mathrm{H} \alpha$ flux along with its eight nearest neighbors (corresponding to a $0.7^{\prime \prime}$ diameter aperture, matching our mean spatial resolution of $\left.0.65^{\prime \prime}\right)$. We assumed this aperture is small enough to probe the inner nucleus part as objects usually span from $1^{\prime \prime}$ to $2^{\prime \prime}$ with $S N R>2$ in our observations.

Figure 2 shows i) the distribution of the 24 galaxies as a function of their global and nuclear N2 ratio (left panel) and ii) the relation between the global and nuclear N2 ratios for each galaxy (right panel). The median values of $\mathrm{N} 2$ for each distribution are

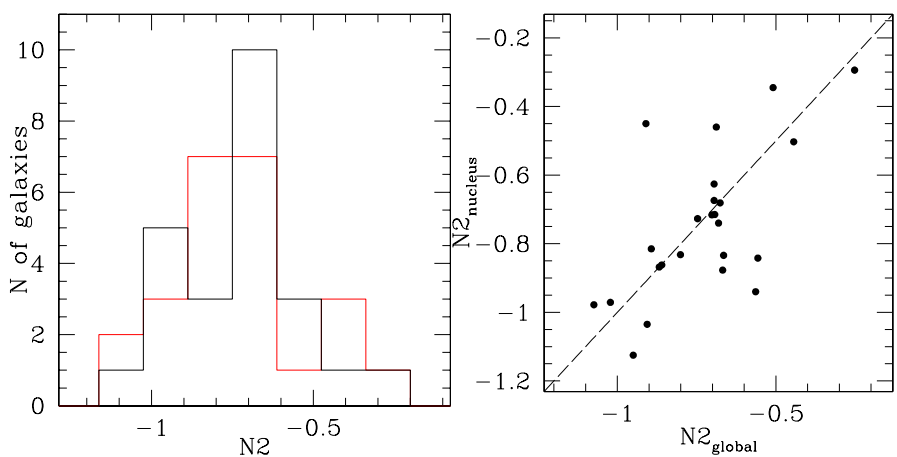

Fig. 2. Left: distribution of the N2 ratio in the MASSIV "first epoch" sample. The black line represents the ratio integrated over the whole galaxy. The red line is the $\mathrm{N} 2$ ratio measured in the nuclear region. Right: N2 global ratio versus N2 nuclear ratio.

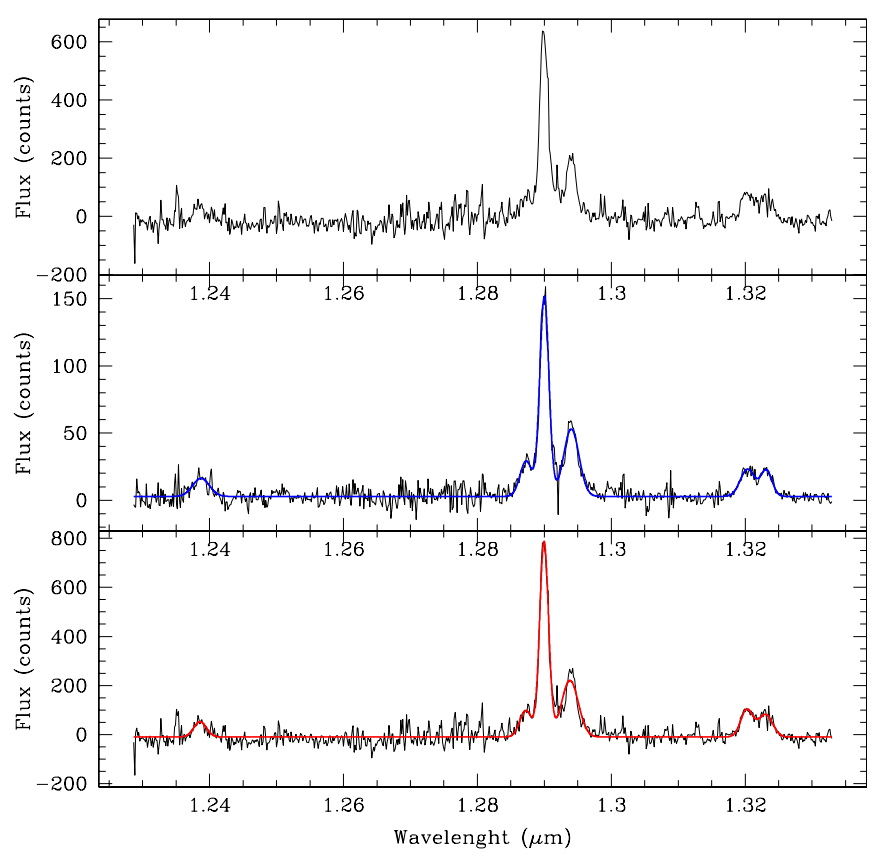

Fig. 3. Integrated spectra of VVDS140096645. Top: spectrum of the disk (total integrated with central contribution removed). Middle: spectrum of the central region surrounding the peak of $\mathrm{H} \alpha$ flux. Bottom: spectrum from the whole spatial extent. From left to right, the following emission lines appear: [O I]6300, [N II]6548, H $\alpha$, [N II]6584, [S II]6717, [S II]6731. The red and blue lines are least-square fits to the data.

not very different $(\Delta \sim-0.07)$. The median nuclear $\mathrm{N} 2$ ratio is lower than the global ratio, which would not be the case if a significant fraction of our sample galaxies were hosting an AGN. When comparing the global ratio to the nucleus ratio distribution, the highest bin does not shift, and it contains a single object. We investigated the galaxy in this bin, VVDS140096645, in more detail. It shows the following high N2 ratios: $N 2_{\text {global }}=$ -0.252 and $N 22_{\text {nuclear }}=-0.294$. Looking further into its integrated spectrum (global and nuclear, see Fig. 3), we noticed that (i) the emission lines are broad, which is a possible sign of nuclear activity; (ii) the two nitrogen lines are clearly visible, as is the sulfur doublet and the [O I]6300 line, which together are characteristics of LINER galaxies, often associated to AGNs (Heckman 1980) - or violent episodes of star-formation in highmetallicity galaxies (Terlevich \& Melnick 1985). 
The galaxy with the next highest global N2 value is $\operatorname{VVDS} 140258511\left(N 2_{\text {global }}=-0.509\right.$ and $\left.N 2_{\text {nuclear }}=-0.345\right)$. Its global and nuclear spectra show no obvious features of nuclear activity, and the $\mathrm{N} 2$ ratio are both $<-0.3$.

We conclude that our sample does not suffer from significant AGN contamination. The only candidate for nuclear activity is VVDS140096645. Similar conclusions were reached based on composite 1D VIMOS and SINFONI spectra of the MASSIV sample (see Contini et al. 2012).

\subsection{Kinematics and close environment classification}

A kinematics and close environment classification of the MASSIV galaxies was performed and is described in detail in Epinat et al. (2012). This classification is based on the $I$-band morphology, the $\mathrm{H} \alpha$ flux maps, the $\mathrm{H} \alpha$ velocity fields, and their modeling assuming a rotating disk.

In the present paper, we exploit two types of classes: a) the dynamical state of galaxies (rotating or non-rotating), and b) the close environment of galaxies. The first class relies i) on the agreement between the position angles of the major axis deduced from the morphology and from the kinematics, and ii) on the accuracy of the rotating disk model. In case the average SNR is lower than 5 , this classification is believed not to be reliable and is thus not used. The second class relies on the detection of companions in both the $I$-band image and $\mathrm{H} \alpha$ maps at a similar redshift to the main source. In some cases, the kinematics maps (velocity fields and velocity dispersion maps) suggest the presence of some physically-related companions or located along the line-of-sight. A confidence flag was assigned to the environment classification, ranging from $\mathrm{A}$ (>90\%, confident) to $\mathrm{C}(\sim 50 \%)$.

We used these classifications to help us constrain the origin of the metallicity gradients in our MASSIV star-forming galaxies.

\subsection{Stellar and dark matter halo masses}

The stellar masses used in our study were obtained with the SED fitting technique. Stellar population synthesis models (Bruzual \& Charlot 2003) were applied to match photometric and spectroscopic data from our MASSIV sample, using the GOSSIP tool (Franzetti et al. 2008). A Salpeter (1955) IMF was assumed. Star formation timescales and the extinction parameter $E(B-V)$ were allowed to range from 0.1 to $15 \mathrm{Gyr}$ and from 0 to 0.3 , respectively. The GOSSIP tool returned the best-fit parameters, as well as a probability distribution function (PDF) for each of them, following Walcher et al. (2008). The median and the standard deviation of the PDF were used to recover the parameter estimates and their associated errors, which in turn were used for this study. The procedure is described in detail in Contini et al. (2012).

The dark matter (DM) halo masses $\left(M_{\text {halo }}\right)$ used hereafter were computed with a spherical virialized collapse model (Peebles 1980; White \& Frenk 1991; Mo \& White 2002):

$$
M_{\text {halo }}=0.1 H_{0}^{-1} \mathcal{G}^{-1} \Omega_{\mathrm{m}}^{-0.5}(1+z)^{1.5} V_{\max }^{3}
$$

where $\mathcal{G}$ is the universal gravitational constant, $z$ the redshift of the galaxy, and $V_{\max }$ the maximum rotational velocity computed in Epinat et al. (2012). To compute associated uncertainties, the Monte Carlo method used in Epinat et al. (2012) for the uncertainties on $V_{\max }$ was extended until the halo mass. The contributions of the two sources of uncertainty on the velocity (inclination and modeling) were added quadratically to compute the final uncertainty.
Table 3. Metallicity and masses.

\begin{tabular}{|c|c|c|c|c|}
\hline Galaxy & (2) & $\begin{array}{r}\Delta_{r} Z \\
{\left[\operatorname{dex~kpc}^{-1}\right]} \\
\text { (3) }\end{array}$ & $\begin{array}{r}\log \left(M^{\star}\right) \\
{\left[M_{\odot}\right]} \\
(4)\end{array}$ & $\begin{array}{r}\log \left(M_{\mathrm{h}}\right) \\
{\left[M_{\odot}\right]} \\
(5)\end{array}$ \\
\hline 020106882 & 8.73 & $0.037 \pm 0.026$ & $9.99_{-0.22}^{+0.22}$ & $11.59_{-0.38}^{+0.20}$ \\
\hline 020116027 & 8.42 & $0.03 \pm 0.024$ & $10.09_{-0.23}^{+0.23}$ & $9.48_{-9.62}^{+0.38}$ \\
\hline 020126402 & & & $10.09_{-0.25}^{+0.25}$ & \\
\hline 020147106 & 8.49 & $0.04 \pm 0.053$ & $10.10_{-0.13}^{+0.13}$ & $9.41_{-12.27}^{+2.87}$ \\
\hline 020149061 & & $\ldots$ & $10.18_{-0.23}^{+0.23}$ & $11.33_{-14.19}^{+2.86}$ \\
\hline 020164388 & 8.58 & $\ldots$ & $10.13_{-0.31}^{+0.33}$ & $10.92_{-071}^{+0.26}$ \\
\hline 020167131 & $\ldots$ & $\ldots$ & $10.08_{-0.20}^{+0.19}$ & $11.58_{-0.61}^{+0.24}$ \\
\hline 020182331 & & $\ldots$ & $10.72_{-0.11}^{+0.11}$ & $11.57_{-0.45}^{+0.22}$ \\
\hline 020193070 & 8.89 & $\ldots$ & $10.15_{-0.20}^{+0.20}$ & $11.52_{-0.42}^{+0.21}$ \\
\hline 020208482 & & & $10.17_{-0.16}^{+0.10}$ & $11.92_{-0.43}^{+0.21}$ \\
\hline 020214655 & 8.76 & $0.0035 \pm 0.015$ & $10.02_{-0.16}^{+0.16}$ & $10.46_{-163}^{+0.30}$ \\
\hline 020217890 & $\ldots$ & $\ldots$ & $9.99_{-0.19}^{+0.19}$ & \\
\hline 020239133 & $\ldots$ & $\ldots$ & 9.8 & $11.84_{-0.56}^{+0.24}$ \\
\hline 020240675 & & $\ldots$ & 9.96 & $10.26_{-13.12}^{+2.87}$ \\
\hline 020255799 & 8.80 & . . & 9.8 & $8.74_{-11.19}^{+2.46^{2}}$ \\
\hline 020261328 & 8.39 & . & 10.0 & $11.41_{-11.45}^{+0.32}$ \\
\hline 020278667 & & & 10.2 & $10.95_{-13.71}^{+2.76}$ \\
\hline 020283083 & 8.74 & $0.045 \pm 0.026$ & 10.0 & $10.56_{-0.46}^{+0.21}$ \\
\hline 020283830 & 8.61 & & $10.37_{-0.17}^{+0.11}$ & $12.02_{-0.30}^{+0.18}$ \\
\hline 020294045 & $\ldots$ & & $9.80_{-0.15}^{+0.15}$ & $12.43_{-0.55}^{+0.23}$ \\
\hline 020306817 & & & $9.76_{-0.2}^{+0.2}$ & \\
\hline 020363717 & 8.57 & $0.0046 \pm 0.039$ & 9.6 & $10.12_{-12.98}^{+2.86}$ \\
\hline 020370467 & 8.75 & $-0.022 \pm 0.054$ & 10.5 & $10.35_{-12.70}^{+2.35^{\circ}}$ \\
\hline 020386743 & 8.53 & $0.0099 \pm 0.024$ & 9.8 & $10.15_{-0.79}^{+0.20}$ \\
\hline 020461235 & 8.78 & $-0.078 \pm 0.023$ & $10.36_{-0.15}^{+0.15}$ & $11.07_{-0.21}^{+0.21}$ \\
\hline 020461893 & $\cdots$ & 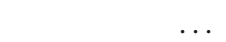 & $9.66_{-0.21}^{+0.21}$ & $10.60_{-0.54}^{+0.23}$ \\
\hline 020465775 & & & $10.12_{-0.20}^{+0.21}$ & $10.72_{-0.54}^{+0.23}$ \\
\hline 140083410 & 8.72 & $-0.02 \pm 0.041$ & $10.07_{-0.18}^{+0.18}$ & $9.77_{-12.18}^{+2.41^{4}}$ \\
\hline 140096645 & 9.23 & & $10.40_{-0.23}^{+0.24}$ & $12.75_{-1500}^{+2.24}$ \\
\hline 140123568 & 8.75 & $0.019 \pm 0.074$ & $9.73_{-0.39}^{+0.39}$ & $10.43_{-13.28}^{+2.850}$ \\
\hline 140137235 & 8.65 & $-0.051 \pm 0.055$ & $10.07_{-0.29}^{+0.29}$ & $10.67_{-0.36}^{-0.19}$ \\
\hline 140217425 & 9.02 & $0.023 \pm 0.0035$ & $10.84_{-0.17}^{+0.19}$ & $12.85_{-0.26}^{+0.16}$ \\
\hline 140258511 & 8.95 & $-0.061 \pm 0.037$ & $10.80_{-0.48}^{+0.48}$ & $11.54_{-0.48}^{+0.22}$ \\
\hline 140262766 & 8.69 & $0.098 \pm 0.11$ & $9.84_{-0.43}^{+0.48}$ & $11.42_{-14.27}^{+2.86}$ \\
\hline 1405 & 8.69 & $-0.033 \pm 0.012$ & 10.6 & $12.23^{+0.24}$ \\
\hline 220014252 & 8.78 & $0.021 \pm 0.0088$ & 10.7 & $11.57^{+0.23}$ \\
\hline 220015726 & 8.75 & $0.00041 \pm 0.02$ & $10.77_{-0.27}^{+0.21}$ & $12.33^{+2.43} .43^{-0}$ \\
\hline 22007 & - 5 & & $10.81_{-0.56}^{+0.27}$ & \\
\hline 220148046 & & & 1 & $10.38_{-1323}^{+2.85}$ \\
\hline 220376206 & 8.40 & $0.04 \pm 0.021$ & & $12.17^{+0.13}$ \\
\hline 220386469 & 8.32 & & 1 & $11.30^{-0.23}$ \\
\hline 220397579 & 8.38 & $-0.022 \pm 0.02$ & & $8.18^{-1.016}$ \\
\hline 220544103 & 8.89 & $0.0063 \pm 0.012$ & & $11.62^{+0.12}$ \\
\hline 220544394 & 8.55 & $0.034 \pm 0.022$ & 10 & $10.54^{+0.21}$ \\
\hline 220576226 & 8.75 & $-0.0073 \pm 0.01$ & & $9.77^{+0.49}$ \\
\hline 220578040 & 8.64 & $0.0031 \pm 0.013$ & 10.72 & $12.48_{-1433}^{+1.86}$ \\
\hline 220584167 & 8.53 & $-0.03 \pm 0.013$ & & $12.31^{+0.13}$ \\
\hline 220596913 & & & & $11.69_{-011}^{+0.08}$ \\
\hline 910193711 & 8.77 & $-0.0091 \pm 0.028$ & & $10.56_{-0.42}^{+0.21}$ \\
\hline 910279515 & 8.60 & (1) & $10.79_{-014}^{+0.18}$ & $12.48_{-0.10}^{+0.08}$ \\
\hline
\end{tabular}

Notes. Column 2: integrated metallicity $(Z=12+\log (\mathrm{O} / \mathrm{H}))$. Column 3: metallicity gradient. Columns 4 and 5: stellar and dark matter halo mass.

Equation (1) makes the assumption that the plateau has been reached and that $V_{\max }$ traces the halo circular velocity. However, this assumption is probably not correct for non rotating systems. The stellar and DM halo masses derived for our galaxies are listed in Table 3. 


\section{Metallicity gradients}

Metallicity gradients in spiral galaxies are commonly measured in the local Universe (Pagel \& Edmunds 1981; Vila-Costas \& Edmunds 1992; Considère et al. 2000; Pilyugin et al. 2004). These gradients are generally negative (metallicity decreasing from the center to the outer parts), and their amplitude is typically $\gtrsim-0.1 \mathrm{dex} \mathrm{kpc}^{-1}$ as traced by the metallicity in $\mathrm{H}$ II regions. In the Milky Way, a gradient of $-0.07 \mathrm{dex} \mathrm{kpc}^{-1}$ is observed.

Several physical processes can be responsible for such gradients (Goetz \& Koeppen 1992). Radial gas flows draining metalrich interstellar gas from the outer parts of the galaxy into the center are believed to play a key role (Tinsley \& Larson 1978). It requires that the infall timescale of gas onto the disk is faster than the star formation timescale. Indeed, Koeppen (1994) has shown that both infall of pristine gas and radial flows into the disk is very efficient in creating an abundance gradient. Phillipps \& Edmunds (1991) further suggested that self-regulating star formation rates varying with the galactocentric distance could generate these gradients. In contrast, for interacting galaxies, Rupke et al. (2010) claim that radial flows of low-metallicity gas from the merging galaxies can explain the low oxygen abundances observed at their center. In these cases, the radial mixing of gas may flatten existing metallicity gradients.

At high redshifts, metallicity gradients are harder to detect because the gas-phase metallicity of individual galaxies can only be measured with limited accuracy. Collisional emission lines from the metals and recombination lines from hydrogen and helium are the only indicators that allow estimation of oxygen abundance in the gas phase, which is needed for a direct comparison with the local galaxies.

In the present study, we used the ratio of the $\left[\mathrm{N}_{\text {II }}\right] 6584$ nitrogen line to the $\mathrm{H} \alpha$ Balmer line as a proxy for the oxygen abundance. Computing this particular ratio has two practical advantages: the [ $\mathrm{N}$ II] 6584 and $\mathrm{H} \alpha$ emission lines are very close in wavelength, so that $i$ ) the differential extinction due to dust attenuation can be neglected; and ii) the relative flux calibration can be considered to be constant over the spectral range. To derive the gas-phase oxygen abundance from this ratio, we used the calibration proposed by Pérez-Montero \& Contini (2009):

$12+\log (\mathrm{O} / \mathrm{H})=9.07+0.79 \times \mathrm{N} 2$

where $\mathrm{N} 2=\log \left(\left[\mathrm{N}_{\mathrm{II}}\right] 6584 / \mathrm{H} \alpha\right)$. This calibration was computed using emission-line objects (star-forming galaxies and $\mathrm{H}$ II regions) in the nearby universe with an accurate oxygen abundance obtained from the measurement of the electronic temperature. The calibration has an intrinsic scatter of $0.34 \mathrm{dex}$, mainly due to the second-order dependence of the N2 parameter on the ionization parameter and on the nitrogen-to-oxygen abundance ratio. As shown in Fig. 4, the PMC09 calibration of the N2 parameter used in this analysis is totally consistent, for both the low- and high-metallicity regimes, with other calibrations of the same parameter found in the literature. Although the N2 parameter was calibrated by PMC09 using data with a determination of $\mathrm{O} / \mathrm{H}$ abundance based only on the "T-method", hence not very well defined for the high-metallicity regime, the PCM09 calibration agrees very well within its dispersion limits (about $0.3-0.4$ dex) with other relations that are partially based on photoionization models or strong-line determinations of the metallicity (Denicoló et al. 2002; Pettini \& Pagel 2004; Nagao et al. 2006). This is especially true in the range of $\mathrm{N} 2$ parameter $(-1.0<\mathrm{N} 2<-0.5)$ covered by MASSIV galaxies (see Table 2 and Fig. 2).

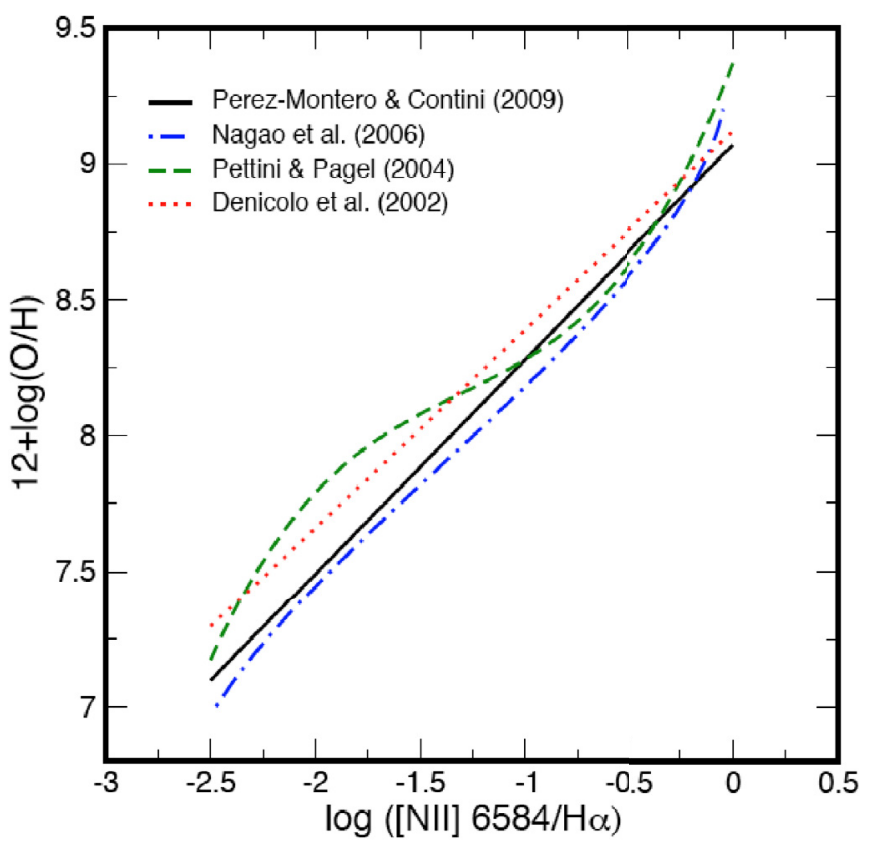

Fig. 4. Comparison between different calibrations used to derive the oxygen abundance from the $\mathrm{N} 2$ parameter. All these calibrations are consistent within their intrinsic dispersion $(\sim 0.3-0.4$ dex $)$, especially in the range of the $\mathrm{N} 2$ parameter $(-1.0<\mathrm{N} 2<-0.5)$ covered by MASSIV galaxies (see Table 2 and Fig. 2).

\subsection{Observed metallicity gradients}

As reported in Sect. 2.2, we measured $\mathrm{H} \alpha$ and $\left[\mathrm{N}_{\mathrm{II}}\right] 6584$ line fluxes in spatially-resolved regions for 26 galaxies. These regions, defined by $\mathrm{H} \alpha$ isoflux contours, are centered on the $\mathrm{H} \alpha$ peak in each galaxy, which most often corresponds to the kinematical center of the galaxy. The widths of the annular regions were adjusted so as to have a high enough $S N R$ for measuring the $\left[\mathrm{N}_{\mathrm{II}}\right] 6584$ line, hence the $\mathrm{N} 2$ ratio. This allowed the radial behavior of the $\mathrm{H} \alpha$ and $\left[\mathrm{N}_{\mathrm{II}}\right] 6584$ lines to be quantified, i.e. of the gas-phase metallicity from the inner to the outer parts of each galaxy.

The derived metallicity gradients and the integrated metallicities are listed in Table 3. The metallicity is estimated as a function of radius, each region having a mean radius and a metallicity (uncertainties on metallicity estimates are dominated by measurements errors). The radius of a region corresponds to the mean radius between its outer and inner contours. The radius of a contour is approximated by the radius of a perfect circle with the same perimeter. Depending on the galaxy inclination, the intrinsic deprojected radius can be underestimated up to a factor of $\sim 1.5$. Metallicities of the different regions in each galaxy are plotted in Fig. 5, along with the best fit line to the data. The figure shows that the detected radial gradients are in general very weak, some being positive and others negative.

Figure 6 (left panel) shows the distribution of metallicity gradients (in dex $\mathrm{kpc}^{-1}$ ) for the 26 MASSIV galaxies, for which we were able to measure [ $\mathrm{N}$ II] 6584 in different regions. The histograms show the full sample, the isolated galaxies, and the interacting ones, as classified in Sect. 2.4. For the last two distributions, we only considered objects classified with a high or medium confidence level (A or B), excluding two isolated galaxies classified with a low confidence level C (VVDS220578040 and VVDS910193711). In the majority of the cases, no clear gradient is detected: the median value of the total sample is 


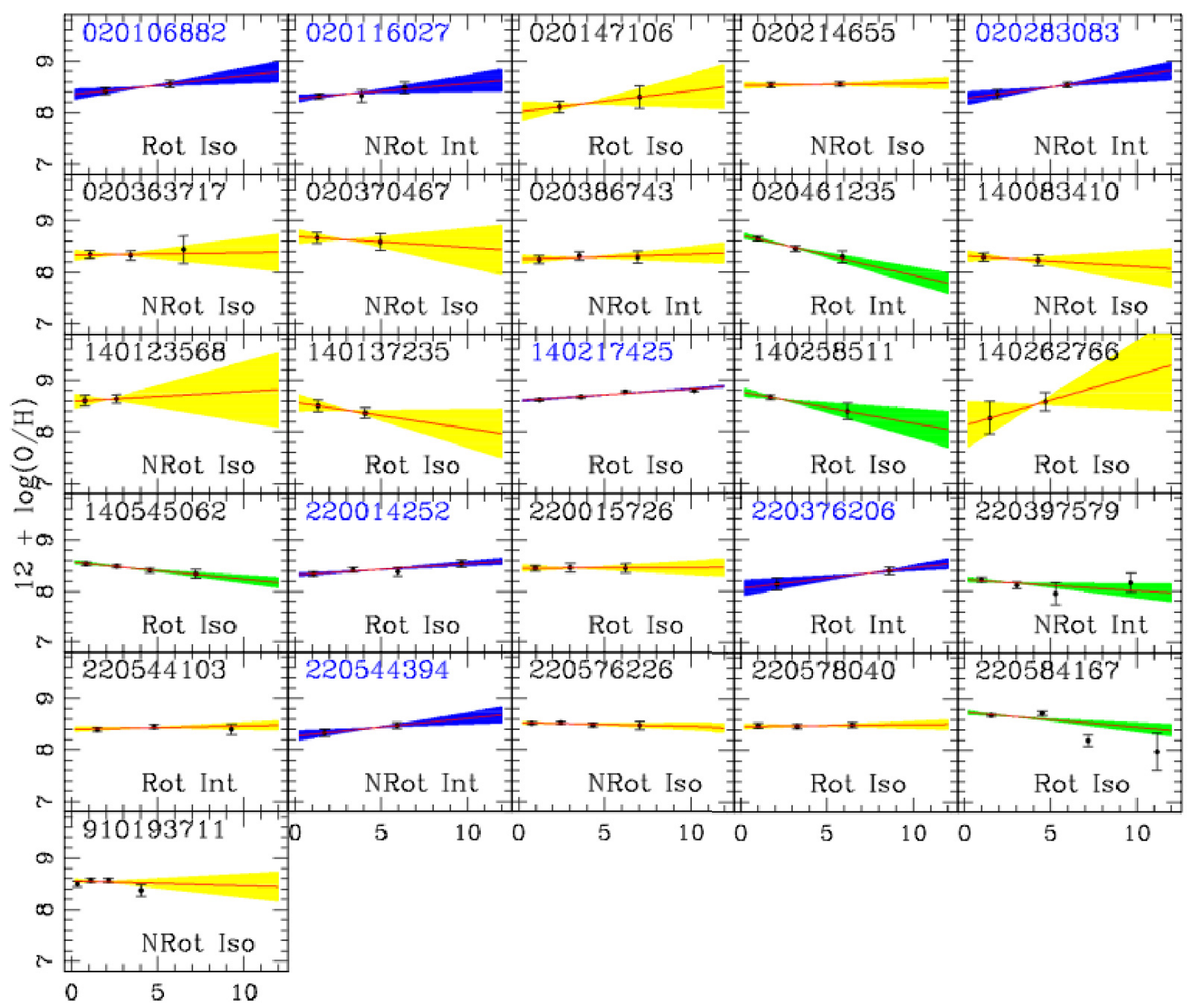

radius $[\mathrm{kpc}]$

Fig. 5. Metallicity gradient for the 26 MASSIV galaxies with spatially-resolved metallicities. The $x$-axis represents the mean radius (in kpc) of the region relative to the $\mathrm{H} \alpha$ center. The $y$-axis indicates the corresponding metallicity in dex $(12+\log (\mathrm{O} / \mathrm{H}))$. The red lines are the best fits to the data, taking the errors on the metallicities into account. The yellow/blue/green regions represent the $1 \sigma$ errors associated to the gradients. Blue label indicates the galaxies for which the gradient is positive within $1 \sigma$, the green ones are those where it is negative within the same limits. For each galaxy we have indicated the dynamical $(\mathrm{Rot}=$ rotating disk, NRot $=$ no rotation) and environment $($ Iso $=$ isolated, Int $=$ interacting $)$ classes .

$0.0040 \pm 0.037 \mathrm{dex} \mathrm{kpc}^{-1}$. The distributions for isolated $(16 \mathrm{ob}-$ jects) and interacting ( 8 objects) galaxies show similar shapes, with median values $0.0018 \pm 0.036 \mathrm{dex} \mathrm{kpc}^{-1}$ for the isolated galaxies, and $0.020 \pm 0.038 \mathrm{dex} \mathrm{kpc}^{-1}$ for interacting ones.

Interestingly, for twelve of the 26 galaxies, a gradient is detected with a $>1 \sigma$ confidence and with nearly the same proportion of positive (seven) and negative (five) gradients (see Fig. 5). Thus, contrary to the global trend in the local Universe, where the gas-phase metallicity of disk galaxies generally decreases with galactocentric radius, seven of our galaxies have higher metallicities in the outskirts than in the center (where the center is defined as the maximum of the $\mathrm{H} \alpha$ flux).

About one fourth of our sample galaxies thus show a positive metallicity gradient at a $>1 \sigma$ confidence level (see Fig. 5). Of these, VVDS220376206 displays a positive gradient with $2 \sigma$ confidence, while in the galaxies VVDS140217425 and VVDS220014252 a positive gradient of $\sim 0.02$ dex $\mathrm{kpc}^{-1}$ is detected with $>5 \sigma$ confidence. Among the seven galaxies, four are classified as interacting systems, while the three others are isolated. One of the isolated galaxies, the secure case VVDS140217425, appears as a "chained-galaxy", with large clumps in the outskirts that could be interpreted as minor mergers. About half (4/8) of the interacting galaxies have a positive metallicity gradient while it concerns only 3/16 ( 20\%) of the isolated ones. We thus tentatively conclude that the majority of the galaxies showing a positive metallicity gradient are interacting. It is interesting to note also that among the four interacting systems, only one galaxy is classified as a rotating disk.

On the other hand, five galaxies display clearly negative metallicity gradients at a $>1 \sigma$ confidence level (of which one is at $>2 \sigma$ and three at $>3 \sigma$ confidence level; see Fig. 5 and Table 3). Among these five galaxies, three are isolated and two are interacting. Four of these five galaxies are classified as rotating disks. 
J. Queyrel et al.: MASSIV: Mass Assembly Survey with SINFONI in VVDS. III.
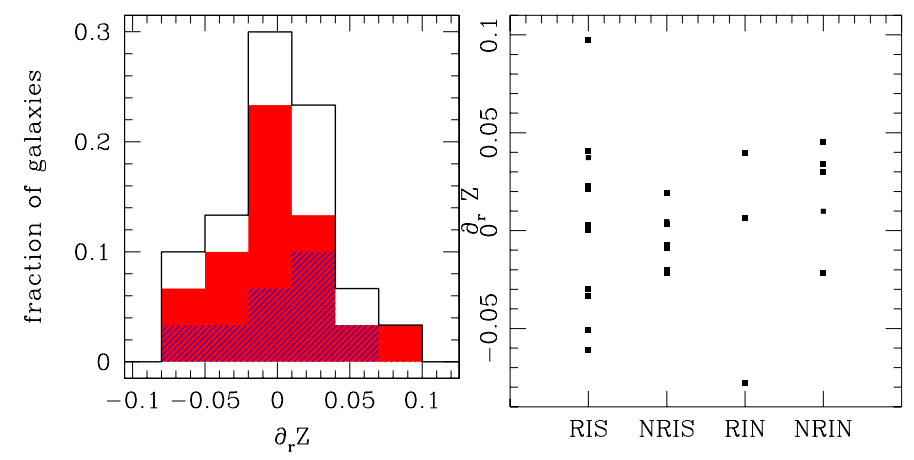

Fig. 6. Left: metallicity gradients distribution for 26 MASSIV galaxies. The histogram in black line represents the whole sample, the red and dashed blue ones the isolated and interacting galaxies distribution respectively. Right: metallicity gradients with respect to the kinematical type (from left to right, rotating isolated, non rotating isolated, rotating interacting, non rotating interacting).

Although rare, this is not the first time that positive gradients of oxygen abundance have been found. Recently, Werk et al. (2010) have reported a positive gradient in a local galaxy and proposed several scenarios to explain their discovery: (i) a radial redistribution of the metal-rich gas produced in the nucleus; (ii) supernovae blowing out metal-rich gas, enriching the IGM, then falling onto the outer parts of the disk; and (iii) the result of a past interaction.

At high redshift, Cresci et al. (2010) have recently studied the metallicity distribution of three Lyman-break galaxies with SINFONI at $z \sim 3$ in the AMAZE/LSD sample. They were able to derive metallicity maps for the three galaxies with high SNR. In each case, they discovered a positive gradient comparable to the ones we have found. They favor the scenario in which these positive gradients are produced by the infall of metalpoor gas into the center of the disks, diluting the gas and lowering its metallicity in the central regions. The authors claim that the discovery of positive gradients in high-redshift disks, pre-selected to be "isolated", is direct evidence of cold gas accretion as a mechanism for mass assembly. Such a conclusion could be balanced by arguing that a merger remnant can keep an inversed metallicity gradient during a transient phase with a typical timescale of $\sim 0.5$ Gyr, (e.g. Perez et al. 2011; Torrey et al. 2012).

In contrast, our study appears to show that, among the seven detected positive gradients, only two galaxies are isolated, the others showing signs of interaction. Cold gas accretion toward the center of disks might thus not be the only process able to lower the central metallicity.

\subsection{Relations with global galaxy properties}

Several authors have found correlations between gas-phase metallicity gradients and other general physical parameters of galaxies. The observed metallicity gradients are a function of the morphological type (Vila-Costas \& Edmunds 1992; Márquez et al. 2002): they are steep in late-type spirals and almost flat for early-type spirals. Furthermore, in the local Universe the absolute value of the gradients seems to decrease with increasing luminosity (less luminous galaxies have steeper metallicity profiles) as predicted by modeling (Prantzos \& Boissier 2000) and verified by observations (Garnett et al. 1997; van Zee et al. 1998).
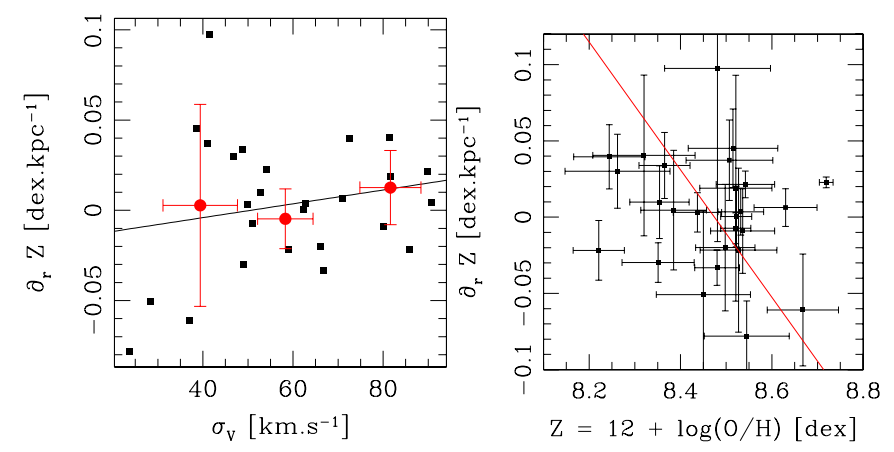

Fig. 7. Left: metallicity gradient versus the mean velocity dispersion of MASSIV galaxies. The black squares are the individual galaxies, and the red ones represent the median values for 3 bins of $\sigma_{v}$, along with the standard deviation in each bin represented by the error bars. Right: metallicity gradients versus the integrated metallicity of each galaxy, with the red line the best fit to the data.

Our high-redshift sample did not verify the last correlations, and we were not able to test a correlation with the morphological type, since we do not have that information for our MASSIV sample. The right hand panel of Fig. 6 shows no clear trend between the strength of the metallicity gradient and the kinematical type, or any correlations with their close environment (isolated or interacting). We can, however, notice that i) among the isolated objects, the non rotating galaxies have on average flatter gradients than rotating disks, and ii) the fraction of positive gradients is higher in interacting systems than in isolated galaxies.

One of the two weak correlations that might be present in our sample is shown in the left hand panel of Fig. 7. The strength of the gradient seems to correlate with the velocity dispersion of the galaxy. The latter is derived on beam-smearing corrected velocity dispersion maps obtained after velocity field modeling (see Epinat et al. 2012) and thus reflects the true velocity dispersion of the gas.

This apparent correlation seems to be driven by the fact that galaxies with high gas velocity dispersion show shallower, often positive, metallicity gradients. This correlation has, to our knowledge, not been observed previously in the local universe, where the velocity dispersion in late-type objects is usually low with $\sigma_{v} \sim 20 \mathrm{~km} \mathrm{~s}^{-1}$ (Epinat et al. 2010). Could turbulent physical conditions in the ISM of high-redshift galaxies be at the origin of the shallow, sometimes positive gradients? This question is difficult to address here, considering the relatively low spatial resolution of our data and the scatter in Fig. 7. At face value, the positive gradients in our $z \sim 1.2$ galaxies might be related to the perturbed physical conditions/motions in the ISM of high- $z$ galaxies, as opposed to the continuous metallicity gradients observed in the relatively quiet ISM of the local spirals.

Finally, we observe as well a tentative anti correlation between the metallicity gradient of each galaxy and its integrated metallicity (see Fig. 7, right panel). Metallicity gradients are more frequently negative in metal-rich galaxies and more frequently positive in low-metallicity galaxies. If real, this behavior would support the scenario in which infall of metal-poor gas from the IGM into the center of the disks drives the positive gradients. This infall of pristine gas would be able to reverse the gradient by diluting the central gas metallicity, while lowering the overall metallicity of the galaxy at the same time. The main question would remain: where does the metal-poor gas come from: accretion of cold gas from the DM reservoir and/or interaction-triggered gas infall/capture from companions? 


\subsection{Gas infall rates}

As already suggested by previous studies (e.g. Werk et al. 2010; Rupke et al. 2010), during an interaction, metal-poor gas from the outskirts of the galaxy could radially flow towards the center and dilute the metallicity in the inner, high star-forming regions. At the same time, metal-enriched gas could be transported to the outer parts during the interaction, resulting overall in a flattening of the metallicity gradient.

The two scenarios explaining the positive metallicity gradients - cold gas accretion and gas redistribution during interactions - have in common that metal-poor gas needs to be transported efficiently to the center of the objects on shorter timescales than the star formation.

We consider the possibility of infall of pristine gas onto the disk in the context of a chemical evolution model in order to explain the positive gradients. Our toy model for the chemical evolution of galaxies assumes (i) an instantaneous recycling approximation (IRA), (ii) the infall of metal-free gas onto the disks, and (iii) radial flows of gas into the disks. This leads to the following equations:

$$
\begin{aligned}
& \frac{\partial g}{\partial t}+\frac{\partial}{r \partial r}(r v g)=-(1-R) \psi(t)+f \\
& \frac{\partial Z}{\partial t}+\frac{\partial}{r \partial r}(r v Z)=y(1-R) \psi(t)-z(1-R) \psi(t)
\end{aligned}
$$

where $g$ is the gas surface density, $\psi$ the star formation rate per unit area, $R$ the "returned fraction", $f$ the infall rate (per unit area) of intergalactic metal-free gas, $Z=z g$ the metal content, $y$ the stellar yield, and $v$ the velocity of the radial gas flow (positive toward the outer parts). Following a Schmidt-Kennicutt law for the star formation rate density $\left(\Sigma_{\psi}=C g^{n}, C=1.6 \times 10^{-27}\right.$, $n=0.7)$, we solve these equations for each galaxies in our sample, assuming typical values for $C, n, y, v$, and $R$ (see above and below). After combining Eqs. (3) and (4), we end up with the following equation allowing us to derive the infall parameter $f$ :

$$
\frac{\partial z}{\partial t}+v \frac{\partial z}{\partial r}=y(1-R) C^{1 / n} \psi^{1-1 / n}-z C^{1 / n} \psi^{-1 / n} f
$$

Assuming the physical parameters involved in Eq. (5) to remain constant for over $\sim 4 \mathrm{Gyr}$ (i.e. valid for galaxies at $z \simeq 1.2$ ), we derive a mean infall rate per unit area that can be integrated since the formation epoch. To this end, we use a true yield value of $y=0.019$, and a returned fraction of $\sim 40 \%: R=0.4$ (Erb 2008). The radial flow velocity of high-redshift galaxies is known to be higher than in local spirals (e.g. $\sim 1 \mathrm{~km} \mathrm{~s}^{-1}$ in the case of the Milky Way). This velocity can be approximated as the velocity towards the center of the migrating clumps - in which the star formation takes place - which then leads to velocities of 10-50 $\mathrm{km} \mathrm{s}^{-1}$ (Bournaud et al. 2007). For our purpose, we used for each MASSIV galaxy the estimated velocity dispersion reported in Epinat et al. (2012).

For the star formation rates, we used those derived from the $\mathrm{H} \alpha$ luminosity, corrected for extinction, and listed in Table 2. Although the $\mathrm{H} \alpha$ luminosity only reflects the instantaneous star formation rate, we assume it to be an average value since the formation epoch. As our galaxies were selected to be star-forming, this is most likely an upper limit on the average star formation.

The calculated global infall rates range from a few to several hundred $M_{\odot} \mathrm{yr}^{-1}$. These values are high compared to the output of hydro-dynamical simulations of typical disk galaxies ( $\left.50 M_{\odot} \mathrm{yr}^{-1}\right)$. However, the rate of infalling gas is believed to depend on the size and mass of the host DM halo, which is able

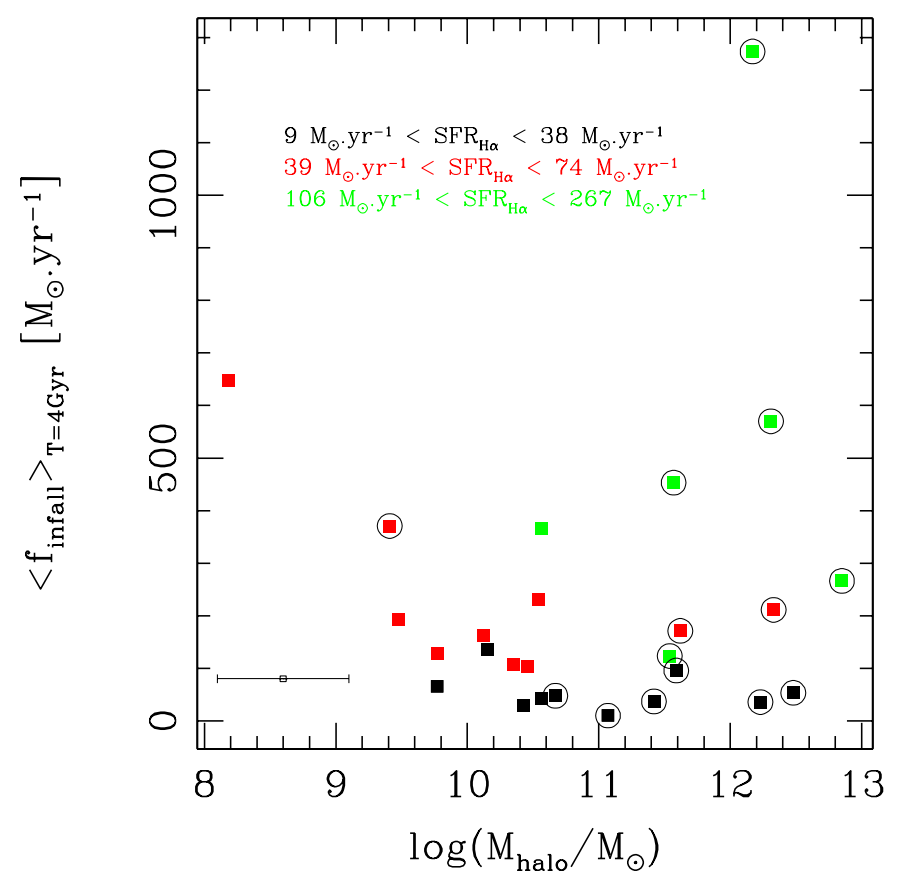

Fig. 8. Mean infall rates over 4 Gyr versus the mass of the hosting DM halo of each MASSIV galaxies. The objects classified as rotators are circled in black. The lower left corner displays a typical errorbar on the DM halo mass.

to reach a few hundred of $M_{\odot} \mathrm{yr}^{-1}$ for the most massive galaxies (Kereš et al. 2005; Erb 2008). We therefore derived DM halo masses for each galaxy in our sample (see Sect. 2.5) and compared it to our calculated infall rates (see Fig. 8). As expected there is a tendency for galaxies to accrete more gas in the most massive halos. We further divided the sample into three bins with respect to the (extinction-corrected) star formation rate. In that case, we noticed that galaxies with the highest SFR also have the highest gas-infall rate, whereas galaxies with the lowest SFR show infall rates typical of local galaxies $\left(\sim 50 M_{\odot} \mathrm{yr}^{-1}\right)$.

Two MASSIV galaxies deviate from the global trend in Fig. 8. VVDS220397579, located at the left end of the graph, has a very low DM halo mass but a high infall rate $\left(\sim 650 M_{\odot} \mathrm{yr}^{-1}\right)$, while VVDS220376206 has by far the highest infall $\left(>10^{3} M_{\odot} \mathrm{yr}^{-1}\right)$ and star formation $\left(>250 M_{\odot} \mathrm{yr}^{-1}\right)$ rates. For VVDS220397579, it is very likely that its DM halo mass was underestimated. Indeed, this galaxy could be a face-on disk because the kinematical modeling returned a very low rotation velocity of $\sim 9 \mathrm{~km} \mathrm{~s}^{-1}$. Since the DM halo mass is directly proportional to the maximum rotation velocity (see Eq. (1)), the DM halo mass for VVDS220397579 is most probably only a lower limit.

In Fig. 8, we have circled those objects that, according to our kinematical classification, are isolated and rotating. For those objects the DM halo mass is more reliable, and they indeed appear to have the highest DM halo masses. To investigate the relationship between the infall rate and the star formation rate, we show in Fig. 9 the mean infall rates against the SFRs corrected for extinction. As expected from Eq. (5), a clear linear correlation between these two quantities can be seen where the infall rates appear to be directly proportional to the star formation rates. The best fit to the data gives a slope of 2.71 for this relation, which is defined as the infall parameter $f_{i}$. This has already been shown to be a typical value for galaxies at these redshifts (Erb 2008). 
J. Queyrel et al.: MASSIV: Mass Assembly Survey with SINFONI in VVDS. III.

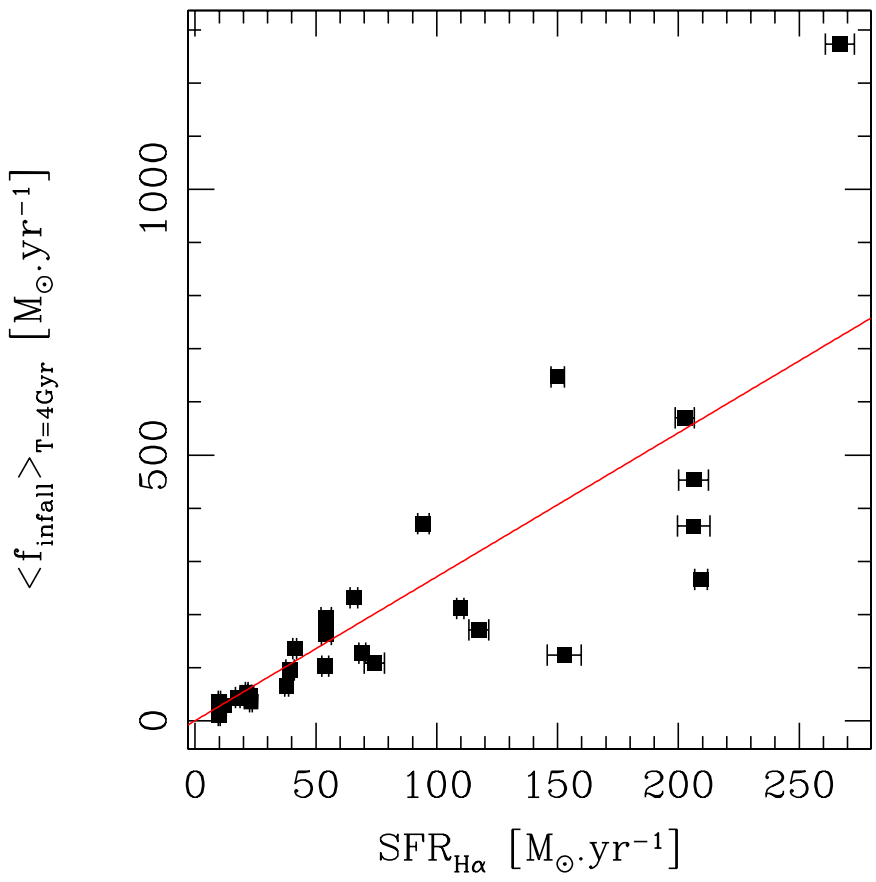

Fig. 9. Mean infall rates versus the star formation rates derived with the $\mathrm{H} \alpha$ luminosity and corrected for extinction. The line is the best fit to the data.

\section{Summary and conclusions}

This paper presents the chemical abundance analysis of the "first epoch" sample (50 galaxies) of the MASSIV survey. Complementary analyses focused on the kinematical classification and the fundamental scaling relations can be found in two companion papers (Epinat et al. 2012; Vergani et al. 2012), as well as a description of the whole MASSIV sample (Contini et al. 2012).

We were able to measure emission lines ([N $\left.\mathrm{NI}_{6}\right] 6584$ and $\mathrm{H} \alpha$ ) in 34 integrated spectra of MASSIV galaxies. Within this sample, we identified one galaxy hosting an AGN. Chemical abundance estimates could be obtained for the remaining 33 starforming galaxies.

For 26 galaxies, we were able to derive a metallicity gradient, thereby defining annular regions around the peak of the $\mathrm{H} \alpha$ flux (which corresponds, in most cases, to the kinematical center). While just over half of our sample is compatible with a zero metallicity gradient, about a quarter of the galaxies show positive gradients where the metallicity increases from the center to the outer parts of the galaxy. Among these last (seven) galaxies, four are classified as interacting systems, one is probably a chain galaxy, and two are classified as isolated.

Flat, more rarely positive, metallicity gradients have already been found in interacting galaxies in the local Universe. They are explained by the infall of metal-poor gas onto the central parts during the encounter (Werk et al. 2010; Rupke et al. 2010).

Two of our galaxies showing a positive metallicity gradient are classified as isolated and do not show any sign of recent interaction. Three analogs objects were reported as isolated disk galaxies at $z \sim 3$ by Cresci et al. (2010). In these cases, cold gas accretion onto the central regions of the disks seems to be the most plausible scenario. Even if tentative, there is a very interesting trend as a function of redshift. At $z \sim 3$ almost all isolated galaxies have a positive gradient, whereas this fraction drops to $\sim 15-20 \%$ at $\sim 1.2$ and is almost equal to zero in the local universe. If cold accretion is indeed the main process to explain the positive metallicity gradients in isolated disks, the epoch where cold accretion dominates the mass assembly processes is at $z \geq 2$.

We noticed in our sample a tendency for galaxies with the highest gaseous velocity dispersion to have a shallow/positive gradient, which highlights the different physical conditions observed in the ISM of high-redshift galaxies (high-velocity dispersion compared to local spirals). We discovered a weak correlation between gradient and global metallicity of the galaxy: metal-poor galaxies usually have flat/positive gradients, while metal-rich ones tend to display negative metallicity gradients. This behavior can also be explained by the infall of metal-poor gas onto the center of the disks, diluting the overall metallicity.

Finally, by applying a simple chemical evolution model with radial flows of gas, we estimated infall rates of pristine gas onto the disks. We found values up to several hundred of $M_{\odot}$ per years, and a tendency for the maximum infall rate to increase with the DM halo mass.

The analysis of the spatially-resolved metallicity of galaxies will be extended to the full MASSIV sample, enabling higher quality statistics, hence a stronger interpretation in terms of galaxy assembly scenarios. The final sample will also help to establish the mass-metallicity and fundamental metallicity relations at $0.9<z<1.8$, adding constraints on the evolutionary status of star-forming galaxies at high redshifts.

Acknowledgements. We wish to thank Léo Michel-Dansac and Frédéric Bournaud for their help and useful comments on this work. We thank also the referee for useful suggestions. This work has been partially supported by the CNRS-INSU and its Programme National Cosmologie-Galaxies (France) and by the French ANR grant ANR-07-JCJC-0009. D.V. acknowledges the support from the INAF contract PRIN-2008/1.06.11.02.

\section{References}

Alonso, M. S., Michel-Dansac, L., \& Lambas, D. G. 2010, A\&A, 514, A57 Baldwin, J. A., Phillips, M. M., \& Terlevich, R. 1981, PASP, 93, 5 Bekki, K., \& Shioya, Y. 1999, ApJ, 513, 108

Bournaud, F., Elmegreen, B. G., \& Elmegreen, D. M. 2007, ApJ, 670, 237 Bruzual, G., \& Charlot, S. 2003, MNRAS, 344, 1000

Considère, S., Coziol, R., Contini, T., \& Davoust, E. 2000, A\&A, 356, 89

Contini, T., Garilli, B., Le Fevre, O., et al. 2012, A\&A, in press (Paper I)

Cresci, G., Mannucci, F., Maiolino, R., et al. 2010, Nature, 467, 811

de Ravel, L., Le Fèvre, O., Tresse, L., et al. 2009, A\&A, 498, 379

Denicoló, G., Terlevich, R., \& Terlevich, E. 2002, MNRAS, 330, 69

Di Matteo, P., Pipino, A., Lehnert, M. D., Combes, F., \& Semelin, B. 2009, A\&A 499, 427

Eisenhauer, F., Abuter, R., Bickert, K., et al. 2003, in SPIE Conf. Ser. 4841, ed. M. Iye, \& A. F. M. Moorwood, 1548

Epinat, B., Contini, T., Le Fèvre, O., et al. 2009, A\&A, 504, 789

Epinat, B., Amram, P., Balkowski, C., \& Marcelin, M. 2010, MNRAS, 401, 2113

Epinat, B., Tasca, L., Amram, P., et al. 2012, A\&A, in press

Erb, D. K. 2008, ApJ, 674, 151

Erb, D. K., Shapley, A. E., Pettini, M., et al. 2006, ApJ, 644, 813

Flores, H., Hammer, F., Puech, M., Amram, P., \& Balkowski, C. 2006, A\&A, 455,107

Förster Schreiber, N. M., Genzel, R., Bouché, N., et al. 2009, ApJ, 706, 1364

Franzetti, P., Garilli, B., Fumana, M., et al. 2008, in Astronomical Spectroscopy and Virtual Observatory, ed. M. Guainazzi, \& P. Osuna, 105

Garilli, B., Le Fèvre, O., Guzzo, L., et al. 2008, A\&A, 486, 683

Garnett, D. R., Shields, G. A., Skillman, E. D., Sagan, S. P., \& Dufour, R. J. 1997, ApJ, 489, 63

Gnerucci, A., Marconi, A., Cresci, G., et al. 2011, A\&A, 528, A88

Goetz, M., \& Koeppen, J. 1992, A\&A, 262, 455

Heckman, T. M. 1980, A\&A, 87, 152

Kereš, D., Katz, N., Weinberg, D. H., \& Davé, R. 2005, MNRAS, 363, 2

Kewley, L. J., \& Dopita, M. A. 2002, ApJS, 142, 35

Kewley, L. J., Heisler, C. A., Dopita, M. A., \& Lumsden, S. 2001, ApJS, 132, 37

Kewley, L. J., Geller, M. J., \& Barton, E. J. 2006, AJ, 131, 2004

Kewley, L. J., Rupke, D., Zahid, H. J., Geller, M. J., \& Barton, E. J. 2010, ApJ, 721, L48 
Kobayashi, C. 2004, MNRAS, 347, 740

Koeppen, J. 1994, A\&A, 281, 26

Lamareille, F., Mouhcine, M., Contini, T., Lewis, I., \& Maddox, S. 2004, MNRAS, 350, 396

Lamareille, F., Contini, T., Brinchmann, J., et al. 2006, A\&A, 448, 907

Lamareille, F., Brinchmann, J., Contini, T., et al. 2009, A\&A, 495, 53

Law, D. R., Steidel, C. C., Erb, D. K., et al. 2009, ApJ, 697, 2057

Le Fèvre, O., Vettolani, G., Garilli, B., et al. 2005, A\&A, 439, 845

Lequeux, J., Peimbert, M., Rayo, J. F., Serrano, A., \& Torres-Peimbert, S. 1979, A\&A, 80, 155

López-Sanjuan, C., Le Fèvre, O., de Ravel, L., et al. 2011, A\&A, 530, A20

Magrini, L., Vílchez, J. M., Mampaso, A., Corradi, R. L. M., \& Leisy, P. 2007, A\&A, 470, 865

Maiolino, R., Nagao, T., Grazian, A., et al. 2008, A\&A, 488, 463

Mannucci, F., Cresci, G., Maiolino, R., et al. 2009, MNRAS, 398, 1915

Márquez, I., Masegosa, J., Moles, M., et al. 2002, A\&A, 393, 389

Michel-Dansac, L., Lambas, D. G., Alonso, M. S., \& Tissera, P. 2008, MNRAS, 386, L82

Mo, H. J., \& White, S. D. M. 2002, MNRAS, 336, 112

Montuori, M., Di Matteo, P., Lehnert, M. D., Combes, F., \& Semelin, B. 2010, A\&A, 518, A56

Nagao, T., Maiolino, R., \& Marconi, A. 2006, A\&A, 459, 85

Pagel, B. E. J., \& Edmunds, M. G. 1981, ARA\&A, 19, 77

Peebles, P. J. E. 1980, The large-scale structure of the universe, ed. P. J. E. Peebles
Peeples, M. S., Pogge, R. W., \& Stanek, K. Z. 2009, ApJ, 695, 259 Perez, J., Michel-Dansac, L., \& Tissera, P. B. 2011, MNRAS, 417, 580 Pérez-Montero, E., \& Contini, T. 2009, MNRAS, 398, 949

Pérez-Montero, E., Contini, T., Lamareille, F., et al. 2009, A\&A, 495, 73 Pettini, M., \& Pagel, B. E. J. 2004, MNRAS, 348, L59

Phillipps, S., \& Edmunds, M. G. 1991, MNRAS, 251, 84

Pilyugin, L. S., Vílchez, J. M., \& Contini, T. 2004, A\&A, 425, 849 Prantzos, N., \& Boissier, S. 2000, MNRAS, 313, 338

Queyrel, J., Contini, T., Pérez-Montero, E., et al. 2009, A\&A, 506, 681 Rupke, D. S. N., Kewley, L. J., \& Barnes, J. E. 2010, ApJ, 710, L156 Salpeter, E. E. 1955, ApJ, 121, 161

Spolaor, M., Proctor, R. N., Forbes, D. A., \& Couch, W. J. 2009, ApJ, 691, L138 Terlevich, R., \& Melnick, J. 1985, MNRAS, 213, 841

Tinsley, B. M., \& Larson, R. B. 1978, ApJ, 221, 554

Torrey, P., Cox, T. J., Kewley, L., \& Hernquist, L. 2012, ApJ, 746, 108

Tremonti, C. A., Heckman, T. M., Kauffmann, G., et al. 2004, ApJ, 613, 898

van Zee, L., Salzer, J. J., Haynes, M. P., O’Donoghue, A. A., \& Balonek, T. J. 1998, AJ, 116, 2805

Vergani, D., Epinat, B., Contini, T., et al. 2012, A\&A, submitted

Vila-Costas, M. B., \& Edmunds, M. G. 1992, MNRAS, 259, 121

Walcher, C. J., Lamareille, F., Vergani, D., et al. 2008, A\&A, 491, 713

Werk, J. K., Putman, M. E., Meurer, G. R., et al. 2010, ApJ, 715, 656

White, S. D. M. 1980, MNRAS, 191, 1P

White, S. D. M., \& Frenk, C. S. 1991, ApJ, 379, 52

Wright, S. A., Larkin, J. E., Graham, J. R., \& Ma, C.-P. 2010, ApJ, 711, 1291 